\title{
Full Stabilization of a Microresonator-Based Optical Frequency Comb
}

\author{
P. Del'Haye, O. Arcizet, A. Schliesser, R. Holzwarth, and T. J. Kippenberg* \\ Max-Planck-Institut für Quantenoptik, 85748 Garching, Germany \\ (Received 11 March 2008; revised manuscript received 20 June 2008; published 31 July 2008)
}

\begin{abstract}
We demonstrate control and stabilization of an optical frequency comb generated by four-wave mixing in a monolithic microresonator with a mode spacing in the microwave regime $(86 \mathrm{GHz})$. The comb parameters (mode spacing and offset frequency) are controlled via the power and the frequency of the pump laser, which constitutes one of the comb modes. Furthermore, generation of a microwave beat note at the comb's mode spacing frequency is demonstrated, enabling direct stabilization to a microwave frequency standard.
\end{abstract}

DOI: 10.1103/PhysRevLett.101.053903

PACS numbers: 42.62.Eh, 42.65.Ky, 42.65.Yj

Introduction. - Optical frequency combs $[1,2]$ have become a powerful tool for high precision spectroscopy over the past decade and are moreover used for various applications such as broadband gas sensing [3], molecular fingerprinting [4], optical clocks [5], and attosecond physics [6]. Frequency comb generation naturally occurs in modelocked lasers whose emission spectrum constitutes an "optical frequency ruler" and consists of phase coherent modes with frequencies $f_{m}=f_{\text {CEO }}+m f_{\text {rep }}$ (where $m$ is the number of the comb mode). Consequently, stabilization of a frequency comb requires access to two parameters: the spacing of the modes, which is given by the rate $f_{\text {rep }}$ at which pulses are emitted, and the offset frequency, given by the carrier envelope offset frequency $f_{\text {CEO }}$, which can be measured and stabilized using the technique of selfreferencing (by employing, for instance, an $f-2 f$ interferometer $[1,7,8])$. Indeed, these techniques have been critical to the success of mode-locked lasers as sources of optical frequency combs.

Recently, a monolithic frequency comb generator has been demonstrated for the first time [9]. This approach is based on continuously pumped fused silica microresonators on a chip, in which frequency combs are generated via parametric frequency conversion through four-wave mixing [10], mediated by the Kerr nonlinearity [11-15]. In this energy-conserving process, two pump photons are converted into a symmetric pair of sidebands with a spacing given by the free spectral range of the microcavity. This four-wave mixing process can cascade and give rise to frequency combs spanning up to $500 \mathrm{~nm}$ in the infrared with a mode spacing of up to $1 \mathrm{THz}$. The comb modes have been shown to be equidistant to a fractional frequency uncertainty of one part in $10^{17}$ relative to the pump frequency [9].

Here we present two major advancements that are necessary preconditions for the monolithic comb generator to be viable in frequency metrology and related applications. First, we demonstrate that it is possible to control the two degrees of freedom of the microcavity frequency comb (MFC) spectrum, which is required for full stabilization of the comb spectrum. In contrast to mode-locked lasers, one comb mode can be directly accessed via the frequency of the pump laser (since it is part of the MFC), whereas the mode spacing as a second degree of freedom is controlled by changing the optical path length of the microcavity via the pump power-dependent refractive index change of the microresonator. This type of comb control is very robust, since no movable elements are involved as in the case of mode-locked femtosecond lasers. The actuators are used to demonstrate full stabilization of a microcavity frequency comb. Second and equally important, we show that smaller mode spacings in the microwave regime $(86 \mathrm{GHz})$ can be achieved using larger scale fused silica toroids with diameters approaching the millimeter range. This result thus overcomes a drawback [16] of our previous work [9], which exhibited a mode spacing in the terahertz range that was not amenable to direct detection with photodiodes. Here we show that these combs produce an amplitude modulation, which is sufficient to directly measure a beat note at the mode spacing frequency. Using this microwave beat note, stabilization of the mode spacing to a frequency standard is demonstrated.

Figure 1(a) depicts a photograph of this next generation of larger scale monolithic comb generators with an increased diameter of $D=750 \mu \mathrm{m}$ and a corresponding
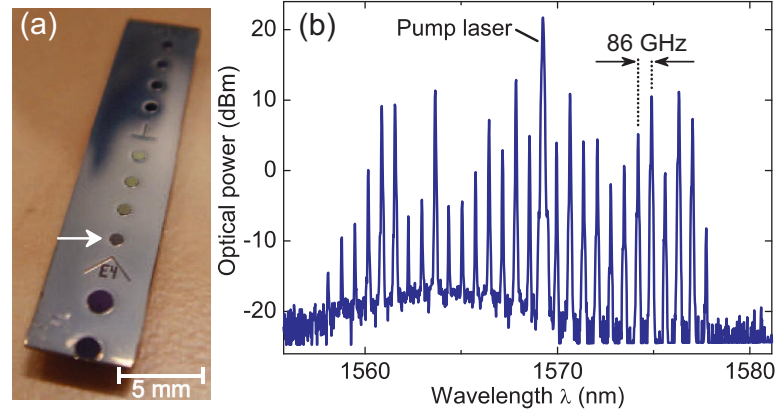

FIG. 1 (color online). (a) Photograph of a silicon chip with a row of $750-\mu \mathrm{m}$-diameter monolithic toroidal microresonators made of fused silica (white arrow). (b) Spectrum of an optical frequency comb, generated by pumping one of the microresonators with $200 \mathrm{~mW}$ laser power (at $1569 \mathrm{~nm}$ ), exhibiting a mode spacing in the microwave frequency range $(86 \mathrm{GHz})$. 
mode spacing of $86 \mathrm{GHz}$ (optical quality factor $Q \approx 2 \times$ $10^{7}$; cavity linewidth $10 \mathrm{MHz}$ ). The measured parametric oscillation threshold of $39 \mathrm{~mW}$ is in good agreement with the theoretical value [11] of $P_{\text {th }} \approx 2 \pi^{2} n^{2} \frac{D A_{\text {eff }}}{\lambda n_{2}} \frac{1}{Q^{2}}$ [17] assuming an effective mode area in the order of $A_{\text {eff }} \approx$ $\pi(2 \mu \mathrm{m})^{2}$ and using, respectively, $n=1.45$ and $n_{2}=2.2 \times$ $10^{-20} \frac{\mathrm{m}^{2}}{\mathrm{~W}}$ for the linear and nonlinear refractive index of silica. The toroids have been made from microfabricated silica disks with an initial diameter of $800 \mu \mathrm{m}$, while the reflow process for the generation of surface tensioninduced toroids has been performed by moving the focus of a $\mathrm{CO}_{2}$-laser beam around the circumference of the silica disks. Coupling of laser light into these resonators is achieved via tapered optical fibers [18] yielding coupling efficiencies of more than 95\%, which is an important prerequisite for high circulating energies within the resonator. Figure 1(b) shows a comb spectrum with $86 \mathrm{GHz}$ mode spacing, produced by pumping a $750-\mu \mathrm{m}$-diameter microtoroid with $200 \mathrm{~mW}$ of continuous wave power at $1569 \mathrm{~nm}$. The spectral width may be improved by increasing the optical quality factor of the microtoroid. Indeed, $Q$ factors of up to $10^{9}$ have been attained in millimeter size microspheres $[19,20]$. Note that the high power per comb mode $(>1 \mathrm{~mW})$ yields direct generation of beat notes with weak laser signals, without using a transfer laser.

Stabilization of the microcavity frequency comb. - The setup to control and stabilize the spectrum of microcavitybased frequency combs is depicted in Fig. 2(a). One mode of a toroidal microresonator is pumped with a tunable external cavity diode laser (ECDL) amplified by an erbium doped fiber amplifier (EDFA), leading to the generation of a frequency comb. In contrast to earlier work [12,21] reporting only phase modulation in the four-wave mixing process, we were able to directly measure the $86-\mathrm{GHz}$ mode spacing beat by sending the comb to a fast photodiode ( $3 \mathrm{~dB}$ cutoff at $50 \mathrm{GHz}$ ). To measure the $86-\mathrm{GHz}$ beat note, it is mixed down to a $30-\mathrm{MHz}$ radio frequency (rf) signal using a harmonic mixer and the 6th harmonic of a local oscillator around $14.3 \mathrm{GHz}$. The generated rf signal (and thus the comb spacing) is stabilized using a phaselocked loop that controls the power launched into the microresonator via the pump diode current of the EDFA.

The second degree of freedom of the MFC is controlled via the frequency of the pump laser (defining a central mode of the comb), which is phase-locked to an optical reference defined by a mode of a fully stabilized erbium fiber laser-based frequency comb with a repetition rate of $\approx 100 \mathrm{MHz}$ [22]. Fast control on the pump laser frequency is achieved by actuation of the diode current of the ECDL (actuation bandwidth $\approx 1 \mathrm{MHz}$ ). Note that this actuator does not affect the launched pump power, since the subsequent EDFA is operated in saturation, amplifying the signal to a constant value. An additional beat note between a MFC mode and an adjacent reference comb mode is used for out-of-loop analysis of the comb stabilization ["beat 2" in Fig. 2(a)]. All local oscillators used for stabilization of
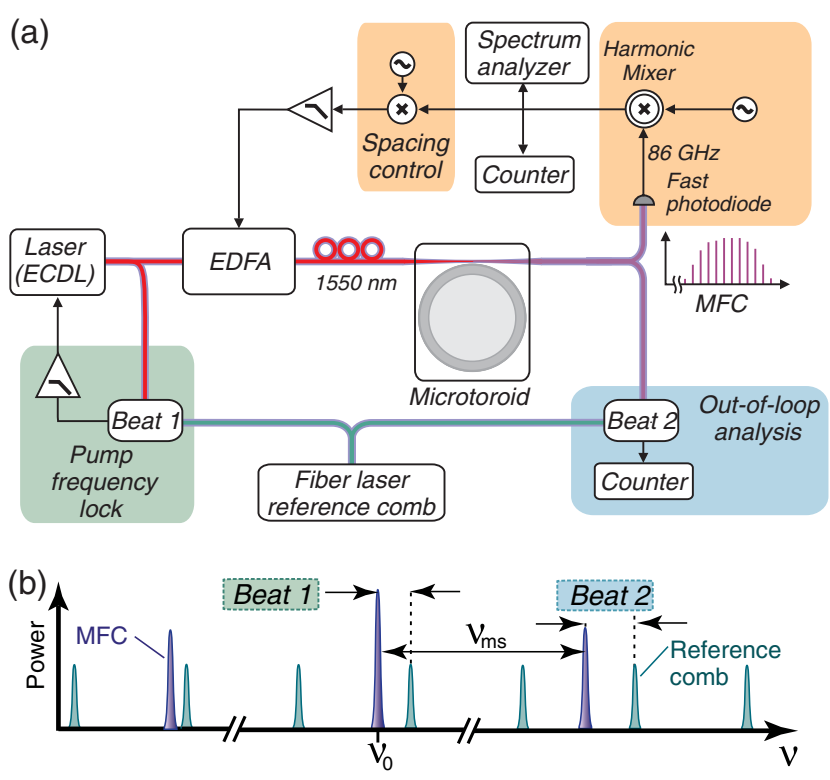

FIG. 2 (color online). Frequency comb stabilization. (a) Scheme of the experimental setup used for stabilizing a microcavity frequency comb. The comb spacing is stabilized via the pump power launched into the microresonator, while the pump frequency is stabilized by a phase lock to a reference comb. (b) Scheme of the microcavity comb and fiber comb modes.

the MFC as well as the fiber laser comb are referenced to the same hydrogen maser.

To obtain a better understanding of the mode spacing of MFCs, a counter measurement of the Allan deviation $\sigma_{A}(\tau)$ of both the stabilized and the free-drifting comb spacing has been conducted (using a Fluke PM6681 counter). At a gate time of $1 \mathrm{~s}$, the free-drifting comb exhibits relative fluctuations of $\sigma_{A}=4.6 \times 10^{-8}$ (corresponding to $4 \mathrm{kHz}$ fluctuations of the $86-\mathrm{GHz}$ carrier), while the stabilized in-loop beat is stable to $\sigma_{A}=$ $7 \times 10^{-13}$ relative to the mode spacing frequency [Fig. 3]. The slow drifts of the mode spacing are attributed to temperature changes, while faster fluctuations are expected to originate from intracavity power fluctuations (arising from pump power fluctuations, coupling instabilities, and competing nonlinear effects within the cavity). The Allan deviation of the counter limit has been measured with a coherent signal at $30 \mathrm{MHz}$, which corresponds to the same frequency as the down-mixed $86 \mathrm{GHz}$ signal, and is also normalized to the $86 \mathrm{GHz}$ mode spacing frequency. The inset in Fig. 3 shows a spectrum of the stabilized microwave mode spacing beat note, whereby the width of the coherent peak is limited by the resolution of the spectrum analyzer $(10 \mathrm{~Hz})$.

Control parameters of the microcavity frequency comb. - Since the pump laser is part of the frequency comb (similar to the comb generators based on intracavity phase modulators [23]), we can describe the comb modes by $\nu_{\ell}=\nu_{0} \pm \ell \nu_{\mathrm{ms}}$ (with the pump frequency $\nu_{0}$, the mode spacing $\nu_{\mathrm{ms}}$, and $\ell$ being an integer number). To measure 


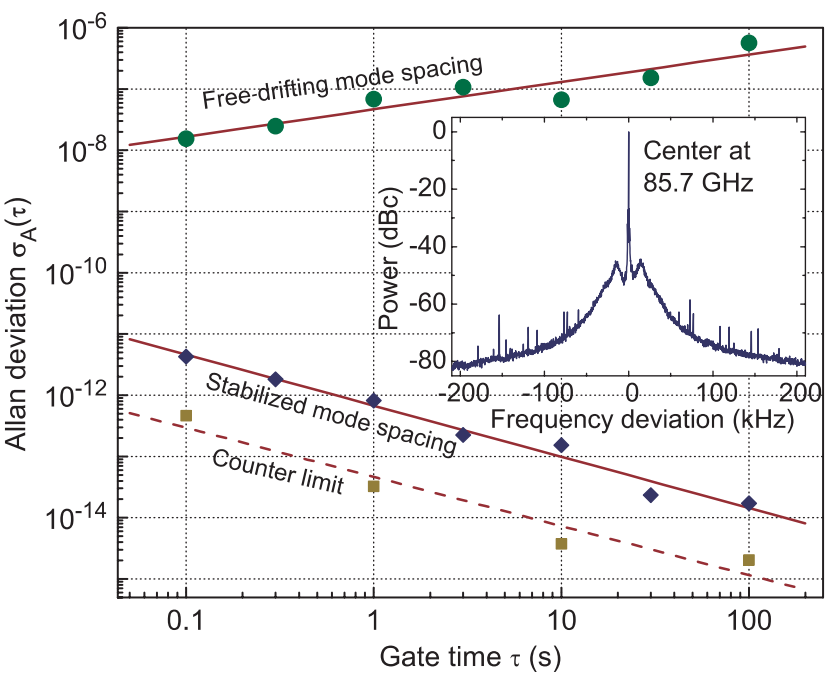

FIG. 3 (color online). Allan deviation of the free-drifting and the stabilized frequency comb spacing. The inset shows a stabilized microwave beat note signal of the mode spacing around $86 \mathrm{GHz}$ with a resolution limited coherent spike (resolution bandwidth $10 \mathrm{~Hz}$ ).

the dependence of the mode spacing $\nu_{\mathrm{ms}}$ on the pump power $P_{\text {in }}$ and pump frequency $\nu_{0}$, one microresonator mode is thermally locked to the pump laser [24] (whereby small fluctuations of the pump detuning are compensated due to the thermally induced frequency shift of the microresonator). This thermal self-locking allows for tuning ranges of tens of gigahertz without losing the resonance of the microcavity [24]. After thermally locking the microcavity mode to the pump laser, we investigate the influence of pump laser frequency $\nu_{0}$ and power $P_{\text {in }}$ on the comb spacing. Using a matrix notation, the two comb parameters can be written as

$$
\left(\begin{array}{c}
\nu_{0} \\
\nu_{\mathrm{ms}}-\nu_{\mathrm{ms}}^{0}
\end{array}\right)=\left(\begin{array}{cc}
1 & 0 \\
\kappa_{\nu} & \gamma_{p}
\end{array}\right)\left(\begin{array}{c}
\nu_{0} \\
P_{\mathrm{in}}
\end{array}\right),
$$

where $\nu_{\mathrm{ms}}^{0}$ is the mode spacing at a certain setpoint used for stabilization. The two parameters $\gamma_{p}$ and $\kappa_{\nu}$ describe the influence of the pump laser's power and frequency on the mode spacing, respectively. They are determined by measuring the mode spacing change when varying the power $P_{\text {in }}$ at a constant frequency $\nu_{0}$ and vice versa. Figures 4(a) and 4(b) show the result of this measurement yielding $\gamma_{p} \approx-20 \mathrm{kHz} / \mathrm{mW}$ and $\kappa_{\nu} \approx 0.65 \mathrm{kHz} / \mathrm{MHz}$ around the chosen setpoint. Thus, having a nonzero $\gamma_{p}$ allows for diagonalization of the transfer matrix in Eq. (1) and consequently independent control of both comb parameters.

The main contribution to the mode spacing change with launched power [cf. Figure 4(b)] can be explained by the changed temperature of the resonator through absorbed optical power. This temperature change leads to a modification of the refractive index of silica which affects the optical path length of the microresonator modes. The thermal contribution has been quantified by comparing the mode spacing tunability with the pump power-

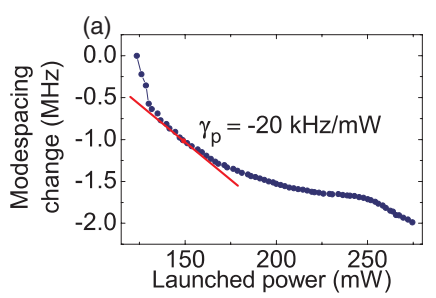

(c)
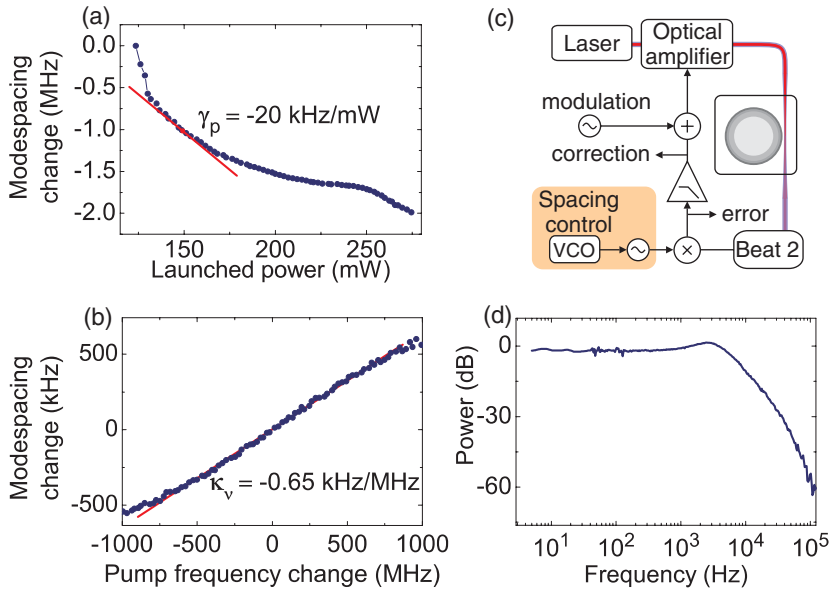

FIG. 4 (color online). (a) Dependence of the mode spacing on the power launched into the microcavity. (b) Dependence of the mode spacing on the pump laser frequency. (c) Experimental setup to determine the locking bandwidth for the mode spacing control. (d) Response measurement of the mode spacing control. The cutoff frequency is around $10 \mathrm{kHz}$.

dependent frequency shift of the microcavity mode. The latter has been measured by scanning the laser over a resonance and measuring the maximum detuning frequency with respect to the cold cavity mode at different powers (see Ref. [24] for details on thermal effects in microcavities). The maximum resonance shift as a function of pump power has been measured to vary with $\Gamma_{p}=$ $-46 \mathrm{MHz} / \mathrm{mW}$, which is in good agreement with the measured mode spacing change multiplied by the mode number, i.e., $\gamma_{p} m \approx-45 \mathrm{MHz} / \mathrm{mW}$ (using $m=2244$ for a cavity mode at $193 \mathrm{THz}$ and a mode spacing of $86 \mathrm{GHz}$ ). Note that for larger pump power changes the mode spacing dependence becomes more complex, which may be attributed to the generation of additional sidebands contributing differently to the thermal effect of the microcavity and the influence of cross- and self-phase modulation [11].

Next the locking bandwidth was investigated using the setup in Fig. 4(c). An external modulation is added to the correction signal of the mode spacing control while simultaneously measuring the correction signal that tries to compensate the perturbation. The result of the measurement in Fig. 4(d) shows the frequency dependence of the correction signal, exhibiting a $3 \mathrm{~dB}$ cutoff at ca. $10 \mathrm{kHz}$. This value is in good agreement with the expected thermal cutoff frequency in silica, which can be estimated by $f_{c}=$ $\frac{\kappa}{2 \pi R_{0}^{2}} \approx 16 \mathrm{kHz}$ [25] (with the thermal diffusivity of silica $\kappa \approx 9 \times 10^{-7} \frac{\mathrm{m}^{2}}{\mathrm{~s}}$ and $R \approx 3 \mu \mathrm{m}$ being the radius of the toroidal cross section). Thus, the small dimensions of the microcavity make the thermal response sufficiently fast for efficient mode spacing stabilization.

Out-of-loop measurements. - To quantify the actual stability of the microcavity comb, an additional out-ofloop beat note of a sideband of the stabilized MFC and the reference comb has been generated and measured. The 


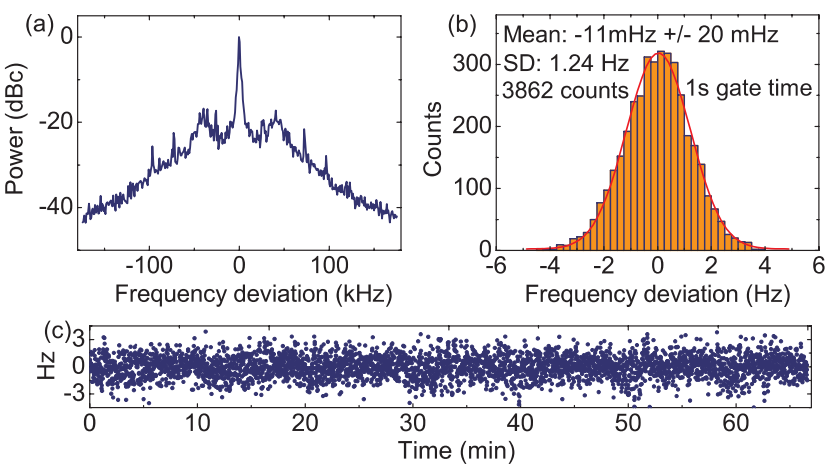

FIG. 5 (color online). Characterization of the stabilized microcavity comb (86 GHz mode spacing). (a) Out-of-loop beat note between a sideband of the stabilized MFC and a fiber laser reference comb (resolution bandwidth $100 \mathrm{~Hz}$ ), measured using the setup in Fig. 2(a). (b) Distribution of the out-of-loop beat note counter measurement data shown below. The deviation from the expected position of the comb line is $-11 \pm$ $20 \mathrm{mHz} . \mathrm{SD}=$ standard deviation. (c) Counter measurement of the out-of-loop beat note at a gate time of $1 \mathrm{~s}$.

stability of the out-of-loop beat note has been measured with a rf counter at a $1 \mathrm{~s}$ gate time and shows a standard deviation of $1.24 \mathrm{~Hz}$ [Fig. 5]. Within the total measurement time of $1 \mathrm{~h}$, this comparison between microcavity comb and fiber laser comb leads to a deviation of $-11 \pm 20 \mathrm{mHz}$ and a relative accuracy of $20 \mathrm{mHz} / 200 \mathrm{THz}=10^{-16}$.

Conclusion.-Stabilization of optical frequency combs generated by four-wave mixing in on-chip microresonators has been demonstrated for the first time. It is emphasized that the presented scheme for stabilization does not require any movable parts and is thus highly mechanically robust. Moreover, generation of microwave beat notes is presented, allowing locking of the mode spacing to a frequency reference. A remaining challenge for selfreferenced frequency comb generators [8] on a chip will be the generation of octave-spanning combs in a microresonator, which could be achieved by improving the optical quality factors and flattening the microcavity's dispersion [26]. The stabilization of a microcavity frequency comb in conjunction with mode spacings in the microwave domain is an important step towards a low cost, small-sized frequency comb generator for spectroscopy applications, astrophysical spectrometer calibration [27], arbitrary optical waveform generation, optical distribution of microwave clock signals [28], and high capacity telecommunication.

We thank T. W. Hänsch for discussions and suggestions. T. J.K. acknowledges support via an Independent Max Planck Junior Research Group. This work was funded as part of a Marie Curie Excellence Grant (RG-UHQ) and the DFG-funded Nanosystems Initiative Munich (NIM). We thank J. Kotthaus for access to clean room facilities for sample fabrication.

\footnotetext{
*tjk@mpq.mpg.de
}

[1] R. Holzwarth, T. Udem, T. W. Hansch, J. C. Knight, W. J. Wadsworth, and P. S. J. Russell, Phys. Rev. Lett. 85, 2264 (2000).

[2] S. A. Diddams, D. J. Jones, J. Ye, S. T. Cundiff, J. L. Hall, J. K. Ranka, R. S. Windeler, R. Holzwarth, T. Udem, and T. W. Hansch, Phys. Rev. Lett. 84, 5102 (2000).

[3] M. J. Thorpe, K. D. Moll, R. J. Jones, B. Safdi, and J. Ye, Science 311, 1595 (2006).

[4] S. A. Diddams, L. Hollberg, and V. Mbele, Nature (London) 445, 627 (2007).

[5] S. A. Diddams et al., Science 293, 825 (2001).

[6] E. Goulielmakis, V.S. Yakovlev, A. L. Cavalieri, M. Uiberacker, V. Pervak, A. Apolonski, R. Kienberger, U. Kleineberg, and F. Krausz, Science 317, 769 (2007).

[7] D. J. Jones, S. A. Diddams, J. K. Ranka, A. Stentz, R. S. Windeler, J. L. Hall, and S. T. Cundiff, Science 288, 635 (2000).

[8] H. R. Telle, G. Steinmeyer, A. E. Dunlop, J. Stenger, D. H. Sutter, and U. Keller, Appl. Phys. B 69, 327 (1999).

[9] P. Del'Haye, A. Schliesser, O. Arcizet, T. Wilken, R. Holzwarth, and T. J. Kippenberg, Nature (London) 450, 1214 (2007).

[10] Optical Processes in Microcavities, edited by R. K. Chang and A. J. Campillo (World Scientific, Singapore, 1996).

[11] T. J. Kippenberg, S. M. Spillane, and K. J. Vahala, Phys. Rev. Lett. 93, 083904 (2004).

[12] A. A. Savchenkov, A. B. Matsko, D. Strekalov, M. Mohageg, V.S. Ilchenko, and L. Maleki, Phys. Rev. Lett. 93, 243905 (2004).

[13] V.S. Ilchenko and A. B. Matsko, IEEE J. Sel. Top. Quantum Electron. 12, 15 (2006).

[14] I. H. Agha, Y. Okawachi, M. A. Foster, J. E. Sharping, and A. L. Gaeta, Phys. Rev. A 76, 043837 (2007).

[15] A. A. Savchenkov, A. B. Matsko, V.S. Ilchenko, I. Solomatine, D. Seidel, and L. Maleki, arXiv:0804.0263.

[16] S. T. Cundiff, Nature (London) 450, 1175 (2007).

[17] The same threshold behavior can be derived by calculating the power that is needed to shift a microcavity resonance by one linewidth via the static Kerr effect.

[18] S. M. Spillane, T. J. Kippenberg, O. J. Painter, and K. J. Vahala, Phys. Rev. Lett. 91, 043902 (2003).

[19] D. W. Vernooy, V. S. Ilchenko, H. Mabuchi, E. W. Streed, and H. J. Kimble, Opt. Lett. 23, 247 (1998).

[20] V. B. Braginsky, M. L. Gorodetsky, and V. S. Ilchenko, Phys. Lett. A 137, 393 (1989).

[21] A. A. Savchenkov, E. Rubiola, A. B. Matsko, V. S. Ilchenko, and L. Maleki, Opt. Express 16, 4130 (2008).

[22] P. Kubina, P. Adel, F. Adler, G. Grosche, T. W. Hansch, R. Holzwarth, A. Leitenstorfer, B. Lipphardt, and H. Schnatz, Opt. Express 13, 904 (2005).

[23] M. Kourogi, K. Nakagawa, and M. Ohtsu, IEEE J. Quantum Electron. 29, 2693 (1993).

[24] T. Carmon, L. Yang, and K. J. Vahala, Opt. Express 12, 4742 (2004).

[25] R. W. Boyd, Nonlinear Optics (Academic, New York, 1992).

[26] V. S. Ilchenko, A. A. Savchenkov, A. B. Matsko, and L. Maleki, J. Opt. Soc. Am. A 20, 157 (2003).

[27] M. T. Murphy et al., Mon. Not. R. Astron. Soc. 380, 839 (2007).

[28] S. M. Foreman, K. W. Holman, D. D. Hudson, D. J. Jones, and J. Ye, Rev. Sci. Instrum. 78, 021101 (2007). 